\section{Findings from the Clinical Information Systems Perspective}

\author{
T. Ganslandt" ${ }^{*}$, W.O. Hackl ${ }^{2 *}$, Section Editors for the IMIA Yearbook Section on Clinical In- \\ formation Systems \\ 1 Medical Center for Information and Communication, Erlangen University Hospital, DE91054 \\ Erlangen, Germany \\ 2 Institute of Biomedical Informatics, UMIT-University for Health Sciences, Medical Informatics and \\ Technology, Hall in Tirol, Austria
}

\section{Introduction}

With the rise of new technologies, methods, tools, tasks and data as well as new user groups and players in the health-IT sector, the field of Health Information Systems (HIS) has become very broad. In addition, the lines between other sections of the IMIA Yearbook of Medical Informatics (especially Decision Support Systems (CDSS), Clinical Research Informatics (CRI) and Human Factors (HF)) and the HIS section risked becoming more and more blurred. Therefore the Yearbook's editorial board decided - starting with the 2015 edition - to narrow the focus of the HIS section on information systems in the clinical domain. Consequently, the section was renamed to Clinical Information Systems (CIS).

Although we defined a completely new query which was intended to be as sharp as possible and included a variety of exclusion criteria in order to rope in the new section, we still retrieved papers which finally had to be assigned to other Yearbook sections. So for example a very interesting paper from Rusanov et al. [1] which indicated possible bias towards the selection of sicker patients when using routine healthcare data from electronic health records for sample selection. This paper had also been in the result set of the CRI section and selected as candidate paper by the CRI section editors (and was finally assigned to this section on mutual consent among the section editors and the editorial board). Another paper by

Yearb Med Inform 2015;10:90-4

http://dx.doi.org/10.15265//Y-2015-037

Published online August 13, 2015
Bouamrane and Mair [2] reporting from the successful implementation of a regional clinical portal for preoperative assessment and an integrated preoperative care pathway and presenting the key success factors for such an implementation was also in the selection of the 2015 Yearbook's special section Patient Centered Care Coordination (PCCC). A variety of other papers which were found by our query could also have been relevant for other sections of the Yearbook. This is quite comprehensible, as CIS have become mature over the last years. The focus has now moved beyond data acquisition for just supporting local care workflows. Breaking down information silos and removing barriers between different systems of different care providers and reusing the accumulated health data for multiple purposes is the obvious actual choice. Thus, many other fields in medical informatics, represented by the different sections of the Yearbook, correlate and sometimes even overlap with the CIS domain.

\section{About the Paper Selection}

Paper selection for the CIS section followed the process described in [3]. Relevant search terms were retrieved first from the Medical Subject Headings (MeSH) catalog, covering major topics from hospital records, health information exchange, health and clinical information systems and meaningful use, among others. The full PubMed query is available upon request from the corresponding author. As indexing with MeSH keywords may occur with a lag of several months after submission to PubMed, ad- 
ditional free text search terms covering the same topics retrieved from MeSH were derived and searched within the title and abstract fields of PubMed. Free-text search was restricted to citations labeled with status "publisher" or "inprocess", which signifies the lack of MeSH-coding. The query was restricted to journal articles published in 2014 in English language and including an abstract. Topics from other IMIA Yearbook sections were excluded with appropriate $\mathrm{MeSH}$ and free text terms. Articles present in the 2014 IMIA Yearbook were excluded. Additional exclusions by publication type were added to restrict the query to original research articles and reviews. The free-text query was additionally reformatted for Web of Science ${ }^{\circledR}$ (from Thomson Reuters) with an additional restriction to the subject area "Medical Informatics".

The query was carried out in mid-January 2015, yielding a combined result set from PubMed and Web of Science of 1525 articles. The resulting articles were published in 722 different journals. Figure 1 depicts the journals with the highest numbers of resulting articles.

The query results were loaded into the BibReview software [3] for a multi-pass review carried out separately by the section editors (TG, WOH), with first-pass selection based on titles and second-pass selection based on abstracts. Results were merged, and articles tagged with pending or conflicting status jointly re-assessed in a final pass, yielding a final selection of 15 candidate papers. These papers were then peer-reviewed by Yearbook editors and external reviewers.

Four papers were selected as best papers for the CIS section (Table 1). A content summary of the 4 selected papers can be found in the appendix of this synopsis.

\section{Findings and Trends: Clinical Information Systems 2014}

In 2014, managing interoperability between different healthcare providers was still a very important task in the field of CIS, and in many cases it is - especially from a syntactical and semantic point of view - still

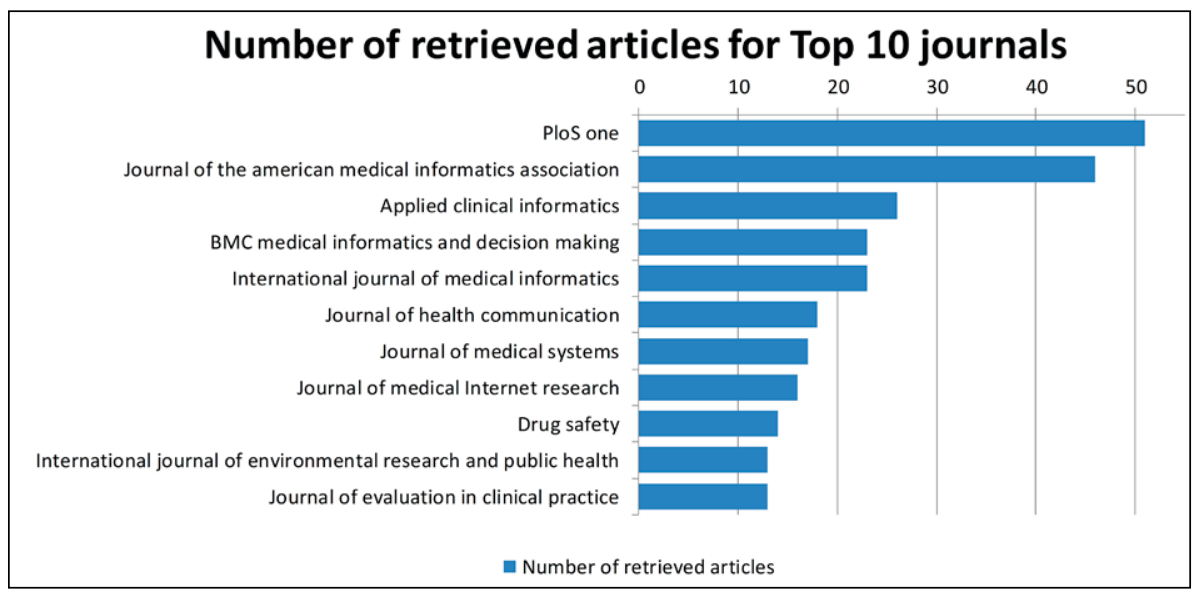

Fig. 1 Top 10 Journals regarding number of retrieved papers resulting from the query for the CIS section.

Table 1 Best paper selection of articles for the IMIA Yearbook of Medical Informatics 2015 in the section 'Clinical Information Systems'. The articles are listed in alphabetical order of the first author's surname.

Section

Clinical Information Systems

- D'Amore JD, Mandel JC, Kreda DA, Swain A, Koromia GA, Sundareswaran S, Alschuler L, Dolin RH, Mandl KD, Kohane IS, Ramoni RB. Are Meaningful Use Stage 2 certified EHRs ready for interoperability? Findings from the SMART C-CDA Collaborative. J Am Med Inform Assoc 2014;21(6):1060-8.

- Li Q, Melton K, Lingren T, Kirkendall ES, Hall E, Zhai H, Ni Y, Kaiser M, Stoutenborough L, Solti I. Phenotyping for patient safety: algorithm development for electronic health record based automated adverse event and medical error detection in neonatal intensive care. J Am Med Inform Assoc 2014;21(5):776-84.

- Meeks DW, Smith MW, Taylor L, Sittig DF, Scott JM, Singh H. An analysis of electronic health record-related patient safety concerns. J Am Med Inform Assoc 2014;21(6):1053-9.

- Plischke M, Wagner M, Haarbrandt B, Rochon M, Schwartze J, Tute E, Bartkiewicz T, Kleinschmidt T, Seidel C, Schüttig H, Haux R. The lower saxony bank of health. Rationale, principles, services, organization and architectural framework. Methods Inf Med 2014;53(2):73-81.

an unsolved challenge. The first of the four selected best papers in the CIS section from D'Amore and colleagues is representative for this finding. This group analyzed 91 C-CDA sample documents from meaningful use stage 2 certified electronic health records (EHRs) and identified a variety of errors as well as permissible heterogeneity that will limit semantic interoperability [4].

Among the retrieved papers we identified different approaches to tackle interoperability problems. Some of them were really striking and two among them were selected as candidate papers for the CIS section. Mandl and colleagues presented a very interesting large scale clinical data research network to enable a queryable semantic data model in the United States [5]. A European contribution by Plischke and colleagues focused more on long-term preservation and organizational/legal issues. In their paper - which finally was selected as one of the four best papers - Plischke et al. introduced the Lower Saxony Bank of Health [6] that implements the concept of Health Record Banking which matches services and architectural frameworks for health information exchange with a business model.

But also smaller initiatives and less complex solutions for health information exchange, semantic integration of different clinical information systems (as for example [2]) or secondary use of existing health data can bring noticeable benefits for health care providers, health professionals and finally the patient. Among the retrieved 
papers we found a large portion of articles dealing with secondary use of health data. Here, many of the contributions dealt with patient safety. The selected best paper from this thematic section came from $\mathrm{Li}$ and colleagues, who presented two algorithms for automatically phenotyping adverse events (AE) and medical errors (ME) in neonatal intensive care [7]. Their algorithms clearly outperformed two standard methodologies in $\mathrm{AE} / \mathrm{ME}$ detection, namely trigger tools and voluntary incident reporting. But also the other candidate papers were very interesting and demonstrated the wide application spectrum of secondary data use for patient safety including, amongst others, real-time automatic surveillance of nosocomial infections [8], prediction models [9] and risk stratification tools [10] for adverse outcomes in hospital wards (e.g. cardiac arrest, ICU transfer, death) or acute kidney injury due to nephrotoxic medication [11].

But, despite all advantages that reusing of integrated health data can bring, we have to keep in mind that the validity of any result gained from these data is directly dependent on the quality of the source data used and no less on the quality of the data integration procedures. Ledikwe and colleagues assessed strengths and weaknesses of health data management and reporting systems in Botswana and concluded that there are many low cost initiatives that can have substantial, positive impact on data management and quality [12]. In their candidate paper [13], Harron and colleagues investigated potential bias due to data linkage errors. They found out that estimates based on linked data can be substantially biased, especially when linkage errors vary by particular groups of records (e.g. records merged from different hospitals due to missing or wrongly recorded identifiers).

Some of the retrieved papers from 2014 led us to conclude that CIS safety concerns persist even after introductory phases. We thus also have to keep in mind that CIS - even though they have reached wide market penetration and are considered a "standard feature" of clinical IT functionality, and besides all the benefits they can bring - hold the potential to harm patients. The last of the four best papers from Meeks and colleagues [14] analyzed EHR-related patient safety concerns and reminds us on this old truth. Although not able to solve this problem, one candidate paper by Price and Lau proposes a very interesting meta-model to at least help describing processes and possible challenges in clinical adoption of health information systems over time [15]. Also not a solution for the problem, but a very interesting compilation of systematically collected barriers and critical success factors for CIS in integrated care settings is presented in the this year's CIS survey by Hoerbst and Schweitzer [16]. There probably never will be a solution to the problem that new developments, technologies and progress in general will sometimes bring unintended and even harmful consequences. Fortunately, the majority of retrieved articles in the CIS section proved the opposite: CIS can positively impact organization and outcomes of health care. The candidate paper by Callen and colleagues [17] who conducted a mixed-method cross-site study on the users' perceptions and impact on change of clinical workflows of an integrated emergency department information system can be representatively mentioned here.

Another old, but likewise important truth is that patients should be involved in their own healthcare. The candidate paper from Otte-Trojel and colleagues [18] reviewing how patient portals can contribute to health service delivery and patient outcomes reminds us of the importance of patient engagement and health literacy. However, when analyzing all the key words from the articles of the CIS section (Figure 2 gives an overview on the Top 10 key words which were used in the retrieved 1525 articles) we could not identify a significant trend towards patient engagement. A trend which perhaps will come to the fore in the next years is the rise of "geographic information systems" which were on rank two according to key words frequency.

\section{Conclusions and Outlook}

Beginning with the 2015 edition of the IMIA Yearbook we narrowed the focus of the former Health Information Systems section to Clinical Information Systems. With a completely new designed query 1525 relevant articles published in 2014 were found. Among these articles we identified two main thematic sections: i) Interoperability from a syntactical and semantic point of view as well as from a long-term preservation and organizational/legal point of view and ii) secondary use of existing health data

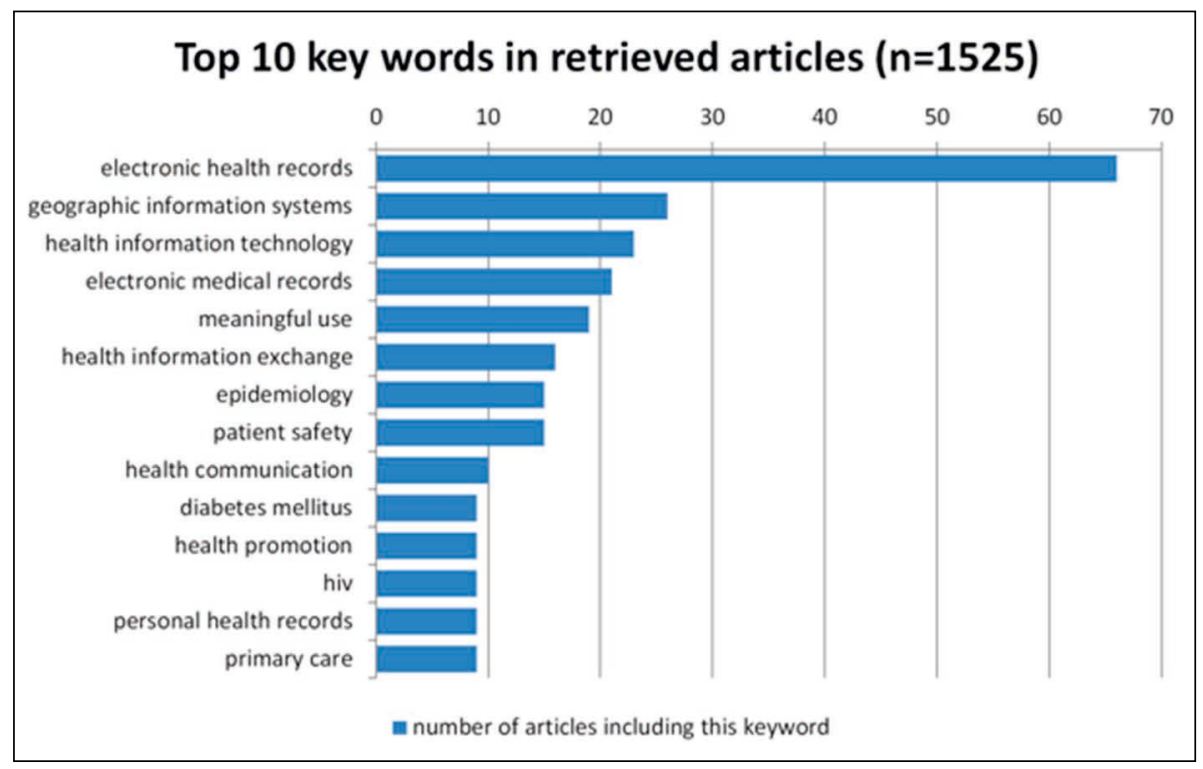

Fig. 2 Top 10 key words (if provided) in retrieved articles ( $n=1525)$ resulting from the query for the CIS section. 
in all its shades. Here, patient safety was a major scope of application. But also besides these two thematic sections we found a lot of very interesting publications reporting from benefits as well as from risks due to CIS. Some of the papers which were found by the 2015 CIS query could also have been relevant for other sections of the Yearbook as they partly correlate or even overlap with the CIS domain. For the next year we will try to reshape the query in order to minimize these overlaps.

\section{Acknowledgement}

We would like to acknowledge the support of Martina Hutter, Chris Lehmann, Marie-Christine Jaulent and Brigitte Seroussi as well as the reviewers in the selection process of the best papers.

\section{References}

1. Rusanov A, Weiskopf NG, Wang S, Weng C. Hidden in plain sight: bias towards sick patients when sampling patients with sufficient electronic health record data for research. BMC Med Inform Decis Mak 2014;14:51.

2. Bouamrane MM, Mair FS. Implementation of an integrated preoperative care pathway and regional electronic clinical portal for preoperative assessment. BMC Med Inform Decis Mak 2014;14:93.

3. Lamy JB, Seroussi B, Griffon N, Kerdelhue G, Jaulent MC, Bouaud J. Toward a formalization of the process to select IMIA Yearbook best papers. Methods Inf Med 2015;54(2):135-44.

4. D'Amore JD, Mandel JC, Kreda DA, Swain A, Koromia GA, Sundareswaran S, et al. Are Meaningful Use Stage 2 certified EHRs ready for interoperability? Findings from the SMART C-CDA Collaborative. J Am Med Inform Assoc 2014 Nov-Dec;21(6):1060-8.

5. Mandl KD, Kohane IS, McFadden D, Weber GM, Natter M, Mandel J, et al. Scalable Collaborative Infrastructure for a Learning Healthcare System (SCILHS): architecture. J Am Med Inform Assoc 2014 Jul-Aug;21(4):615-20.

6. Plischke M, Wagner M, Haarbrandt B, Rochon M, Schwartze J, Tute E, et al. The lower saxony bank of health. rationale, principles, services, organization and architectural framework. Methods Inf Med 2014;53(2):73-81.

7. Li Q, Melton K, Lingren T, Kirkendall ES, Hall E, Zhai H, Ni Y, et al. Phenotyping for patient safety: algorithm development for electronic health record based automated adverse event and medical error detection in neonatal intensive care. J Am Med Inform Assoc 2014 Sep-Oct;21(5):776-84.

8. Du M, Xing Y, Suo J, Liu B, Jia N, Huo R, et al. Real-time automatic hospital-wide surveillance of nosocomial infections and outbreaks in a large Chinese tertiary hospital. BMC Med Inform Decis Mak 2014;14:9.

9. Churpek MM, Yuen TC, Park SY, Gibbons R, Edelson DP. Using electronic health record data to develop and validate a prediction model for adverse outcomes in the wards*. Crit Care Med 2014 Apr;42(4):841-8.

10. Churpek MM, Yuen TC, Winslow C, Robicsek AA, Meltzer DO, Gibbons RD, et al Multicenter development and validation of a risk stratification tool for ward patients. Am J Respir Crit Care Med 2014 Sep 15;190(6):649-55.

11. Kirkendall ES, Spires WL, Mottes TA, Schaffzin JK, Barclay C, Goldstein SL. Development and performance of electronic acute kidney injury triggers to identify pediatric patients at risk for nephrotoxic medication-associated harm. Appl Clin Inform 2014;5(2):313-33.

12. Ledikwe JH, Grignon J, Lebelonyane R, Ludick $\mathrm{S}$, Matshediso E, Sento BW, et al. Improving the quality of health information: a qualitative assessment of data management and reporting systems in Botswana. Health Res Policy Syst 2014;12:7.

13. Harron K, Wade A, Gilbert R, Muller-Pebody B, Goldstein H. Evaluating bias due to data linkage error in electronic healthcare records. BMC Med Res Methodol 2014;14:36.

14. Meeks DW, Smith MW, Taylor L, Sittig DF, Scott JM, Singh H. An analysis of electronic health record-related patient safety concerns. J Am Med Inform Assoc 2014;21(6):1053-9.

15. Price M, Lau F. The clinical adoption meta-model: a temporal meta-model describing the clinical adoption of health information systems. BMC Med Inform Decis Mak 2014;14:43.

16. Hoerbst A, Schweitzer M. A systematic investigation on barriers and critical success factors for Clinical Information Systems in integrated care settings. Yearb Med Inform 2015;10:79-89.

17. Callen J, Li L, Georgiou A, Paoloni R, Gibson K, Li J, et al. Does an integrated Emergency Department Information System change the sequence of clinical work? A mixed-method cross-site study. Int J Med Inform 2014 Dec;83(12):958-66.

18. Otte-Trojel T, de Bont A, van de Klundert J, Rundall TG. How outcomes are achieved through patient portals: A realist review. J Am Med Inform Assoc 2014;21(4):751-7.

Correspondence to

Dr. Werner 0 Hackl

Institute of Biomedical Informatics

UMIT - University for Health Sciences, Medical Informatics and Technology

Eduard-Wallnoefer-Zentrum 1

6060 Hall in Tirol, Austria

Tel: + 435086483806

E-mail: werner.hackl@umit.at

Dr. med. Thomas Ganslandt

Medizinisches IK-Zentrum

Universitätsklinikum Erlangen

Glückstr. 11

DE-91054 Erlangen, Germany

Tel + 49 9131 85-36712

E-mail: thomas.ganslandt@uk-erlangen.de
Appendix: Content Summaries of Selected Best Papers for the IMIA Yearbook 2015 Section "Clinical Information Systems"

D'Amore JD, Mandel JC, Kreda DA, Swain A, Koromia GA, Sundareswaran S, Alschuler L, Dolin RH, Mandl KD, Kohane IS, Ramoni RB

Are Meaningful Use Stage 2 certified EHRs ready for interoperability? Findings from the SMART C-CDA Collaborative

\section{J Am Med Inform Assoc 2014;21(6):1060-8}

Interoperability between care providers has been the focus of the recent "Meaningful Use" incentive program in the United States, mandating support for the exchange of electronic health record (EHR) information based on the Consolidated Clinical Document Architecture (C-CDA). While this standard should enable automated processing of clinical data through defined syntactic structures and permissible terminologies, research into previous comparable standards has shown that implementation issues can preclude successful interoperability.

In this paper, d'Amore et al. collected 91 sample C-CDA documents from 19 vendors and subjected them to manual and automated analysis to identify common trouble spots. Multiple warnings, errors and heterogeneities were found in all documents examined, including omission of XML elements, internal inconsistencies and terminology misuse. While some problems could be detected automatically with existing tools, or could potentially be automatically detected with further programming (e.g. use of deprecated terminologies), many issues would require manual inspection.

The authors provide recommendations for improving C-CDA document quality, including the provision of richer sample documents, the inclusion of terminology code validation by the EHR certification bodies, the reduction of data optionality and the continuous tracking of document quality e.g. as an added service of health information exchange.

The authors conclude that while the Meaningful Use initiative has led to signif- 
icant progress towards interoperability, current C-CDA documents used for EHR data exchange will omit important clinical information and require manual reconciliation.

\section{Meeks DW, Smith MW, Taylor L, Sittig DF, Scott JM, Singh H}

An analysis of electronic health recordrelated patient safety concerns

\section{J Am Med Inform Assoc 2014;21(6):1053-9}

While clinical information systems can improve the efficiency and safety of patient care, they have also been shown to introduce novel safety concerns due to usability issues, disruptions of workflows or unintended workarounds created by users to circumvent system constraints. Combined socio-technical approaches are required to detect, analyze and prevent errors that may arise both from unsafe technological features as well as user behaviors and organizational factors.

In this paper, Meeks et al. describe a retrospective analysis carried out over the "Informatics Patient Safety" (IPS) voluntary incident reporting system established at the United States Veterans Affairs hospital system since 2005. A total of 100 consecutive investigations over a 3 year period were manually analyzed and categorized both into 8 sociotechnical dimensions as well as 3 phases of electronic health record (EHR) implementation and use.

From analysis and interpretation of the categorized results, 4 major types of safety concerns emerged. Most common were unmet data display needs, in which the data provided is insufficient to complete the required task, failing to reduce uncertainty or leading to patient harm. The second most common concern related to intended or unintended software modifications, including upgrades or configuration mistakes leading to failures. The third most common concern related to system-system interfaces, including the failure to synchronize patient context between applications running side-by-side or the disruption of network services e.g. required for remote allergy checks. The final concern related to hidden dependencies in distributed systems, in which complex process interactions can lead to unintended consequences (e.g. the automated removal of inpatient med- ication orders when a patient was temporarily placed into an outpatient unit).

The authors conclude that EHR installations should be systematically monitored for errors, and that comprehensive approaches covering both technical as well as sociological dimensions are required.

\section{Li Q, Melton K, Lingren T, Kirkendall ES, Hall E, Zhai H, Ni Y, Kaiser M, Stoutenborough L, Solti I}

Phenotyping for patient safety: algorithm development for electronic health record based automated adverse event and medical error detection in neonatal intensive care

\section{J Am Med Inform Assoc 2014;21(5):776-84}

With the increasing amount of data acquired in clinical information systems, automated detection of adverse events (AE) and medical errors (ME) becomes a viable secondary use scenario. In this context, phenotyping is defined as the use of algorithms to detect cohorts of patients with AE or ME.

In this paper, Li et al. report on a project to algorithmically detect selected AE/ME in a neonatal intensive care unit (NICU) in comparison to voluntary incident reporting and an existing trigger-tool approach, validated against a manual chart review. The authors additionally describe the manual annotation of a NICU dataset for additional AE/MEs.

An algorithm was developed to detect intravenous infiltrates through the documentation of treatment with hyaluronidase, a substance whose main indication is for i.v. infiltrates. The algorithm outperformed both the existing trigger tool as well as voluntary reporting. A second algorithm was developed to detect several $\mathrm{AE} / \mathrm{ME}$ conditions with opioid treatment. Oversedation was detected by the documentation of treatment with naloxone, whose main indication is to counteract opioids. Opioid dosing errors were detected by comparison to local dose standards as well as analysis of dosing escalation. The algorithm detected more AE/MEs than the trigger tool and voluntary reporting, but also delivered false positives due to documentation errors and small dosing discrepancies that were not clinically relevant.
The authors conclude that algorithmic detection can identify previously undetected or underreported adverse events and medical errors in a neonatal intensive care scenario.

Plischke M, Wagner M, Haarbrandt B, Rochon M, Schwartze J, Tute E, Bartkiewizz T, Kleinschmidt T, Seidel C, Schüttig H, Haux R

The lower saxony bank of health.

Rationale, principles, services, organization and architectural framework

\section{Methods Inf Med 2014;53(2):73-81}

Health Record Banking (HRB) has been proposed to foster the long-term availability and exchange of clinical information between healthcare providers. In this paper, Plischke et al. give a comprehensive overview of the structure, technical architecture and governance aspects of the Lower Saxony Bank of Health (LSBH) in Germany.

The LSBH was founded as an entrepreneurial company, funded by participating healthcare providers (e.g. hospitals) and acting as a neutral third party. Its basic principles include the storage of patient records at the originating healthcare providers (rather can centralized storage), the primary role of the patients to authorize access to their medical data, and the importance of transparency, trust, verifiability and quality in the implementation of HRB services. The technical architecture provides distinct service layers for transport (communication infrastructure, archiving and backup), administration (master patient index, document registry, authorization, provider directory, auditing and gateways) and functions (registering documents, sending structured documents and images to collaborating providers). Implementation will be based on IHE standards (Integrating the Healthcare Enterprise), including XDS (cross-enterprise document sharing), EMPI (enterprise master patient index), XCA (cross-community access) and ATNA (audit trail and node authentication).

While the LSBH remained a work in progress at the time of publication, the authors posit that the chosen structure and architecture matches the specific requirements of the German health care system and data protection legislation. They conclude that organizational barriers may outweigh technical challenges in the further implementation of the LSBH. 